\title{
Improving the quality of weekend handover at Yeovil District Hospital
}

Sarah Sneller, Karolina Lada, Charlotte Turner, Sinead Millwood, Bethan Jervis, Julia Barr, Louise Farrell

Yeovil District Hospital

\begin{abstract}
"Handover of care is one of the most perilous procedures in medicine" (British Medical Association, Safe Handover, Safe Patients). The system in place for weekend handover at YDH was deemed disorganised, unstructured and frequently missing key pieces of information, leaving the on-call Foundation Year 1 (FY1) doctor with only vague jobs and management plans. Baseline surveys demonstrated that junior doctors felt the system was inadequate, potentially compromised patient safety and increased their stress levels.

In order to improve this problem a structured weekend handover proforma was created, comparable with the "Out of hours handover record keeping standards: template" from the Royal College of Physicians. This was made readily accessible on the local intranet. Education sessions were organised for the FY1 and FY2 doctors. The impact of the newly introduced proforma was measured using feedback surveys each week from the FY1 on ward cover for six months. A further change implemented was the introduction of a Friday Ward Round proforma. The aim was to reduce the time required to review notes by the on-call doctor, to minimise avoidable weekend jobs and to improve compliance with the management plans.
\end{abstract}

The results demonstrated $100 \%$ compliance with the new proformas. There were notable improvements in the presence of a plan (37.5\% to $91.7 \%$, max. $100 \%$ ), a minimum of two patient identifiers (68.8\% to $100 \%)$ and relevant background information (62.5\% to $100 \%)$. Qualitative data showed a much higher level of satisfaction with the new system.

Future plans include rolling out electronic handover to improve problems such as illegible handwriting and missing data (enable 'compulsory' fields), and also for this system to be implemented Trust-wide.

\section{Problem}

The handover of patient care has been described as one of the most perilous procedures in medicine. Professor Sir John Lilleyman from the National Patient Safety Agency describes this as "a major contributory factor to subsequent error and harm to patients" in a recent document produced by the British Medical Association (BMA). The junior doctors working in Yeovil District Hospital (YDH) echoed these concerns regarding the weekend handover system.

The current system required the ward doctors to compile a list of jobs for the on-call Foundation Year 1 (FY1) doctor. This was usually handwritten on a blank piece of paper and left for collection in one of the medical wards on a Friday evening. The junior doctors reflected on their experiences and felt that a lack of structure lead to an inconsistency amongst each handover and often a lack of detail. A typical example of a job being handed over was:

"Ward 4, Mrs Bloggs: UTI, check bloods". Due to a lack of adequate information (eg patient identifiers, relevant past medical history) it was often very difficult to initiate a plan to complete each job. It was felt that this had often compromised the safety of patients, was an increased source of junior doctor stress levels, and led to additional hours being worked. It was therefore decided to address this issue with the fundamental aim of improving patient care.

\section{Background}

The General Medical Council states that it is our duty to contribute to the safe handover of patients.[2] The care of a patient may be handed over between several different clinical teams throughout even a short hospital stay. This is of paramount importance during a weekend where staffing levels are reduced for two or more consecutive days. Indeed, studies have shown a significant increase in mortality of patients admitted at the weekend.[3,4] The British Medical Association have issued guidance for junior doctors in creating a safe handover of their patients in recognition of our changing working patterns.[1] Despite this, poor quality of weekend handover has been identified as a contributing factor to significant adverse events.[5]

At Yeovil District Hospital, the medical team is reduced to just four or five doctors over the weekend. This small team is led by the medical registrar who is joined by either one or two senior house officers (SHOs), and Foundation Year 1 trainee (FY1) to clerk and care for all new admissions. They are largely based in the emergency department and medical admissions unit. There is one "ward cover" FY1 to attend all other medical inpatients in the hospital, covering five medical wards and their outliers in addition to the coronary care and high dependency units. The role of the ward cover FY1 involves the completion of jobs handed over from the medical doctors each Friday, in addition to responding to new requests from each ward. The FY1 will rarely be familiar with the patients in question. In reviewing an unwell patient, or chasing the result of an investigation, the doctor will be reliant on information that has been handed over or will alternatively need to undertake a 
detailed review of the medical notes.

\section{Baseline measurement}

Qualitative and quantitative data was obtained to provide baseline measurements for this project $(n=7)$. First, a survey was sent to all FY1s and those FY2s who had done a medical job as an FY1 at Yeovil District Hospital. The survey included ranking on a five-point scale the level of satisfaction with the presence of sufficient patient identification, relevant background, the diagnosis/primary issue, the weekend job, and a management plan. The second section of the survey enquired (based on a five-point scale) to what extent the FY1/FY2 agreed with a series of statements. These included questions about the content of the handover, whether they felt it compromised patient safety, their stress levels, and overall satisfaction and quality regarding the handover sheet.

Our results showed that $100 \%$ of FY1s and FY2s were unsatisfied with the current handover. Specifically, 29\% were dissatisfied with the job itself, 29\% were dissatisfied with the patient identifiers, $86 \%$ were dissatisfied with the main problem/diagnosis, $100 \%$ were dissatisfied with the background, and importantly, $86 \%$ were dissatisfied with the plan. The results of the second section revealed that $86 \%$ believed the current handover contributed to their stress levels and $100 \%$ had to work additional hours to compete their handover jobs. $71 \%$ of respondents agreed that they spent a disproportionate amount of time reviewing patients' notes, $86 \%$ disagreed or strongly disagreed that they had a clear idea of what the plan was for each job, $100 \%$ either disagreed or neither agreed/disagreed that they were provided with sufficient handover information and $29 \%$ agreed that the majority of requests made were inappropriate. It also revealed that $71 \%$ of foundation doctors felt that the current handover compromised patient safety. One respondent commented, "jobs often marked as 'check bloods' with no idea what to do if things were abnormal. Difficult to read people's handwriting."

Based on subjective data, the baseline survey clearly showed dissatisfaction with the weekend handover in its current format. Quantitative data was also collected by analysing the content of the handover sheets over two weekends $(n=15)$. The main problem areas identified were a lack of patient identifiers $(30 \%)$, lack of background (22\%), and lack of a plan (53\%).

In summary, the baseline surveys demonstrated that there was universal agreement amongst junior doctors that the current handover was inadequate, potentially compromised patient safety, and increased their stress levels.

See supplementary file: ds3497.pdf - "Baseline Measurement Data"

\section{Design}

The first intervention was to standardise the handover sheets given to the on-call FY1. A proforma was devised consisting of a table containing the minimum essential information believed to be required for each patient that was handed over (please see attachment under Strategy - "PDSA cycles and handover proforma"). This was comparable to the template produced by the Royal College of Physicians.[9] The proforma specifically had a column for 'Job' and a separate column for 'management plan' in response to feedback from junior doctors as this was most frequently inadequate and a particular source of frustration.

The format for this proforma was a universally compatible word processing document which was uploaded to the Trust intranet. This made it easily accessible for all doctors from any Trust computer. An additional benefit of this format was that the proforma could be downloaded and filled in electronically then printed, or printed blank so the doctor could complete it by hand.

A session was held to educate colleagues about the project, each section of the handover proforma was discussed, and how it could be accessed. This was also used as an opportunity to receive their feedback about the proforma before implementation.

This handover proforma has been designed with the intention that it should be used as a basis for an electronic handover system in the near future. Collaboration with the IT department has led to the production of prototype systems. The prototype allows the on-call FY1 to easily prioritise and categorise jobs (eg by date, by type of job), to remove them from the list once they've been completed (stored in a "completed" section), and eliminates the "messy" factor of carrying around stacks of loose pieces of paper thus also offering better patient confidentiality. It is felt that e-handover may be more sustainable in the long-term especially as the Trust is moving in the direction of more electronic methods, eg vital observations charts, eprescribing, and an e-handover would be compatible with this system.

The second intervention was another education session run by a senior house officer two months after the proforma was introduced. On the basis of weekly handover analysis, we found that not all sections of the handover sheets were always filled out. Therefore, the aim of the education session was to remind the juniors to fill in all of the categories, to provide further clarification about the appropriateness of weekend jobs and to answer any further questions regarding the new handover.

The third intervention was the introduction of a Friday ward round sheet. This was based on a project done by Dr Victoria Cordell and Dr Sarah Kipling (FY1 doctors at Royal United Hospital Bath NHS Trust). The aim was to encourage junior doctors to plan for the weekend when reviewing patients on their existing ward round, and to reduce the amount of time it takes the FY1 on call to review patients' notes at the weekend. The summary sheet contained space to fill in the Friday ward round review with a separate space to document a clear weekend plan. It also contained reminders regarding Friday jobs, such as reviewing the resuscitation status, ensuring the drug chart was re-written in anticipation should it run out over the weekend, and completing the warfarin chart. This sheet has proven to be useful to both the on-call FY1 and other ward team members for viewing the current plan for patients who may unexpectedly require medical attention over the weekend. It will require input from both the juniors and administration teams (such 
BMJ Quality Improvement Reports

as the ward clerks) to remain sustainable. Further feedback from junior and senior doctors as well as nursing staff will be obtained regarding this intervention.

\section{Strategy}

Please see attachment "PDSA cycles and handover proforma".

See supplementary file: ds3596.pdf - "PDSA cycles and handover proforma"

\section{Post-measurement}

Method

There are five medical wards in Yeovil District Hospital often with two teams per ward providing a weekend handover. A sample of four handed-over patients per medical team handover sheet was analysed every weekend and marked 'Yes' or 'No' on the basis of whether eight different criteria were met. The patients were chosen by the first and last patient on each handover sheet (if two handover sheets were provided by one ward), or the first two and last two patients (if just one handover sheet from a ward was provided). Data were collected from the 22nd of November to the 13th of April. Some weekends were missed due to the handover sheets being misplaced by the weekend on-call team.

In addition to the quantitative date, a reflective survey was carried out with the on-call FY1 after every weekend. The aim was to collect data on whether they felt they had a clear management plan for each patient, what time they finished work, whether they had time for a break, the overall quality of the handover, and any further suggestions or comments.

Results

Following the introduction of the proforma, the amount of handovers containing the minimum number of patient identifiers improved and despite a small drop there has been incremental improvement compared with the baseline data. Percentage of handovers containing background information about the patient also improved and increased further following our second and third PDSA cycles.

Prior to introducing our weekend handover, less than $50 \%$ of the handovers had a clear plan for the patient. Since introducing the proforma and running an education session at least $80 \%$ of handovers now have a plan. Data from the reflective survey showed an appreciable increase in the satisfaction with the presence of a clear plan. During the final few weeks of the project, $100 \%$ of the handovers were consistently marked as "top quality".

The comments received from junior doctors about the new handover sheet were very positive, in particular with reference to the plan section. One comment stated "[it was] Extremely useful to have a standardised handover sheet with clear plans and relevant background information about the patient, especially compared to the blank sheets we used before". Please see the supplementary file attached.

See supplementary file: ds3513.pdf - "Results"

\section{Lessons and limitations}

There were a number of challenges along the way. There was some resistance initially from one of the FY1 doctors on a medical ward. That said, the team otherwise engaged the juniors very well and the uptake was generally excellent. Despite designing proformas to fill in, there were occasionally problems with unclear handwriting and not all the fields being filled in. An e-handover that incorporates compulsory fields prior to submitting would address both these problems.

It was difficult to objectively measure patient safety. Ideas such as liasing with the outreach team, counting the number of incidents and using the MEWS scoring over the weekend presented with their own difficulties. Furthermore, there are a number of extraneous variables influencing patient safety that cannot be accounted for. This means that questions about patient safety were incorporated into the surveys and subjective data was collected as a representative measure.

Informal positive feedback was received from doctors and, unexpectedly, even nurses on the Friday ward round sheet after it had been implemented. However, formal evaluation of this cycle of the project has not yet been performed.

At the time of writing, one of the team members printed and distributed the forms each Thursday. In order for this to be sustainable, the ward clerks and IT department will need to be fully involved. In addition, the team will ensure the new take of doctors in August 2014 are well informed about the project. This will be achieved by including it in the 'Essential information for new doctors' session run by the outgoing FY1s, and during the formal shadowing period to ensure they are educated about how to use the forms.

The project was run within the general medicine department. The other departments each have their own methods to hand over patients for the weekend, however it was felt that the handover proforma created for medical handover has potential to be implemented with equal success in other departments, such as surgery and orthopaedics. To achieve good compliance elsewhere, further education would be required.

The future aim would be to roll-out an e-handover. A prototype was designed but due to IT staff limitations this was not fully completed. As Yeovil Hospital moves towards e-healthcare records (including electronic prescribing), the aim would be to incorporate the handover into this system. E-handover has several advantages including reduction of paper usage thereby being more environmentally friendly, potentially reduce problems with patient confidentiality being compromised, and lead to overall greater efficiency.

\section{Conclusion}


BMJ Quality Improvement Reports

An evident problem was identified with the poor quality of medical handovers at the weekend in Yeovil Hospital, demonstrated clearly by qualitative and quantitative baseline measurements. Errors through human factors (such as spending excessive time reviewing notes, deciphering handwriting, incorrect patient identification) could be minimised by the presence of standardised methods. Therefore a handover proforma was developed and implemented and its efficacy was measured on a weekly basis using subjective survey data and quantitative analysis of the information present on all of the medical handover sheets.

The results demonstrate increased satisfaction among junior doctors using the new handover proforma. Handovers are now more likely to contain the appropriate number of patient identifiers (70-100\%), relevant background (62-100\%), and perhaps most importantly, a clear management plan (40-92\%). The latter was noted by members of the Exeter QIA conference, where positive feedback was received on the uniqueness of including an independent section for a management plan. In addition, Friday ward round summaries have been implemented which, on the basis of preliminary multidisciplinary feedback, have optimised time spent reviewing notes by the on-call FY1, and has also benefited the nursing staff.

It is felt that this project has improved patient safety, despite challenges in its objective measurement. A high level of junior doctor satisfaction was achieved with ultimately complete engagement. On the basis of success so far, future plans include development of e-handover and implementation in other departments.

\section{References}

1. British Medical Association . Safe Handover, Safe Patients: Guidance on Clinical Handover for Clinicians and Managers. British Medical Association; 2004.

2. General Medical Council. Good Medical Practice. General Medical Council; 2013.

3. Aylin P; Yunus A; Bottle A; Majeed A; Bell D. Weekend mortality for emergency admissions. A large, multicentre study. Qual saf Health care; 2010.

4. Dr Foster Intelligence: Hospital Guide; 2011. Accessed via: http://drfosterintelligence.co.uk.

5. Pfeffer PE, Nazareth D, Main N, Hardoon S, Choudhury $A B$. Are weekend handovers of adequate quality for the oncall general medical team? Clin Med 2011;11(6): 536-40.

6. Curtis $\mathrm{O}$, Fisher R. Improving medical $\mathrm{SHO}$ weekend handover at a tertiary referral centre. BMJ Qual Improv Rep 2013;2.

7. Mehra A, Henein C.Improving hospital weekend handover: a user-centered, standardised approach. BMJ Qual Improv Rep 2014;2:2.

8. Ashton C. Improving weekend patient handover. BMJ Qual Improv Rep 2013;2:2.

9. Royal College of Physicians. Acute care toolkit 1: handover. https://www.rcplondon.ac.uk/resources/acute-caretoolkit-1-handover.

\section{Declaration of interests}

Nothing to declare.

\section{Acknowledgements}

We would like to thank Dr Jim Gotto, Jo Howarth, the audit department at Yeovil District Hospital, in particular Daniel Gibbs and Charlotte Mitchell. We would also like to thank Dr Victoria Cordell and Dr Sarah Kipling for sharing their Friday ward round summary proforma. 